\title{
Optimal Design of Grooved Cam Profile Using Non-uniform Rational B-splines
}

\author{
Guantao Xuan ${ }^{1,2}$, Yuanyuan Shao ${ }^{1, *}$, and Lei $\mathrm{Liu}^{1}$ \\ ${ }^{1}$ College of Mechanical and Electronic Engineering, Shandong Agricultural University, 271018 Tai'an, China \\ ${ }^{2}$ Shandong Province Key Laboratory of Horticultural Machinery and Equipment, 271018 Tai'an, China
}

\begin{abstract}
In order to reduce the fatigue damage in grooved cam mechanisms, grooved cam profile was reconstructed with non-uniform rational B-splines (NURBS). Considering joint friction, dynamic model of grooved cam mechanisms was established and the contact stress between grooved cam and follower was calculated using Hertz contact theory. Taking the minimum contact stress and the minimum acceleration as optimal objectives, integrated design model for respective kinematic and dynamic design approaches was set up. The integrated design mode was optimized to search Pareto-optimal solution by an improved artificial fish swarm algorithm, and optimized NURBS profile for grooved cam was acquired. The results show NURBS profile has better kinematic and dynamic performances. The impacts on grooved cam mechanism are reduced and wear characteristics are improved.
\end{abstract}

\section{Introduction}

As a kind of cam mechanisms, grooved cam mechanisms are widely used in various automatic machines, such as assembly manipulator, packing and agricultural machinery, etc. Mechanical properties of grooved cam mechanisms are concerned in industrial practices and hence frequently studied in literature [1-3]. The contact stress at the cam-follower interface is larger due to the line-contact higher pair, which leads to contact fatigue wear, influence the working performance, even failure. The lager acceleration also exaggerates the shaking forces and moments, which results in vibrations, poor motion precision, noises and fatigue damage. Recently, there are a series of studies to improve the mechanical properties of cam mechanisms by optimizing the structure parameters and using excellent motion curves such as polynomial curve [4], spline curve [5-6], nonuniform rational B-splines [7-8] and other curves, but all these researches are rarely related to grooved cam mechanisms.

In this paper, non-uniform rational B-splines was adopted to reconstruct the grooved cam profile and dynamic model of grooved cam mechanisms was established. Considering the kinematic and dynamic integration method, multi-objective optimization model of grooved cam mechanisms was set up for reducing the peak value of the contact stress and acceleration. An optimization simulation example was given to compare NURBS profile with traditional cam profile in the end.

\section{Grooved cam mechanisms dynamic analysis}

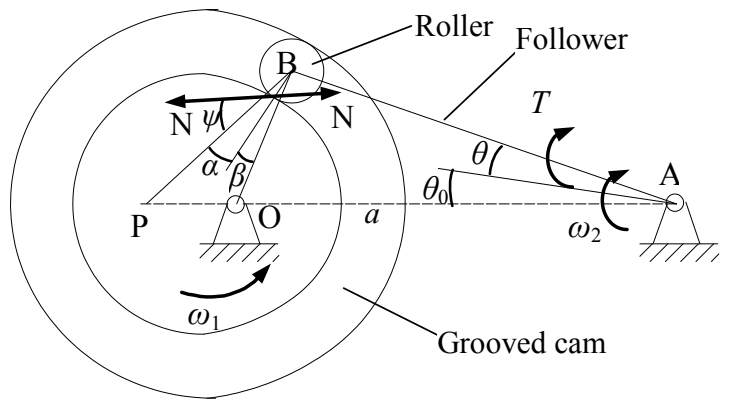

(a) Same direction of rotation $(\mathrm{m}=-1)$

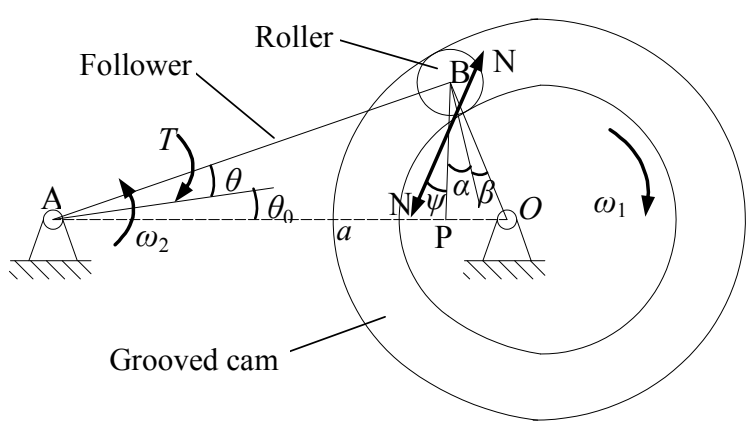

(b) Different direction of rotation $(\mathrm{m}=1)$

Fig. 1. Mechanical model of grooved cam mechanism

Corresponding author: xoyo111@163.com 
Grooved cam mechanisms can be regarded as mechanical model shown in Fig. 1. Where, O, A is the centre of grooved cam and the oscillation centre of the follower respectively, the length of $\mathrm{OA}$ is $a$, the center of the roller is $\mathrm{B}$, length of the follower $\mathrm{AB}$ is $l$, radius of the roller is $r_{0}, \theta$ and $\varphi$ is the angular displacement of the oscillating follower and the grooved cam respectively. Groove cam rotational speed $\omega_{1}$ can be defined as $\omega_{1}=d \varphi / d t$, the oscillating follower angular velocity $\omega_{2}$ be defined as $\omega_{2}=d \theta / d t, \mathrm{P}$ is the instantaneous center of the oscillating follower and the groove cam. Applying Kennedy Aronhold the orem to grooved cam mechanisms, Equation (1) and (2) are deduced as follows.

$$
\begin{aligned}
& \overline{O P}=\frac{a \omega_{2}}{\omega_{1}+m \omega_{2}}=\frac{a \frac{d \theta}{d \varphi}}{1+m \frac{d \theta}{d \varphi}} \\
& \overline{A P}=\frac{a \omega_{1}}{\omega_{1}+m \omega_{2}}=\frac{a}{1+m \frac{d \theta}{d \varphi}}
\end{aligned}
$$

In Equation (1) and (2), $m=-1$ when the cam rotates in the same direction with the oscillating follower as shown in Fig. 1 (a); else, $m=1$ as shown in Fig. 1 (b).

Refer to Fig. 1, let $r_{b}$ denote the radius of base circle, and $\theta_{0}=\arccos \left(\frac{a^{2}+l^{2}-r_{b}^{2}}{2 a l}\right)$ is the initial angle. The pressure angle $\alpha$ at the contact point can be derived from Equation (1) and (2).

$$
\alpha=\arctan \left(m \frac{a \cos \left(\theta_{0}+\theta\right)-l\left(1+m \frac{d \theta}{d \varphi}\right)}{a \sin \left(\theta_{0}+\theta\right)}\right)
$$

In Fig. 1, considering frication of rotary pair and motion pair in grooved cam mechanisms, resultant force $N$ can be derived by applying torque balance principle.

$$
N=\frac{T}{l \cos (\alpha+\psi)+m r_{o} \sin \psi+m\left(\rho_{1}-\rho_{2}\right)}
$$

Where $\psi=\arctan f$, which is angle of friction between the grooved cam and the oscillating follower, $T$ is external torque exerted on the follower, $f$ is the friction coefficient, $\rho_{1}, \rho_{2}$ represent the radius of friction circle of the roller and the oscillating follower rotating center A respectively.

For grooved cam mechanisms with oscillating follower, there is line contact between the grooved cam and the roller in the theory. But contact surface is actually a tiny rectangular contact region due to the elastic deformation of the material when load is imposed on grooved cam mechanisms. According to Hertz contact theory, the peak value of the contact stress occurs in the mid-line of contact region, contact stress $\sigma_{H}$ is defined as follows.

$$
\sigma_{H}=\sqrt{\frac{F_{N}}{\pi b} \cdot \frac{\frac{1}{R_{c}}+\frac{1}{R_{f}}}{\frac{1-\mu_{c}^{2}}{E_{c}}+\frac{1-\mu_{f}^{2}}{E_{f}}}}
$$

In Equation (5), $F_{N}=N \arccos \psi$ is normal pressure of the grooved cam pair, $b$ is the length of contact line between grooved and roller, $\mu_{c}, \mu_{f}$ are Poisson ratio of material for grooved cam and roller, and $E_{c}, E_{f}$ are elastic modulus of material for grooved cam and roller respectively. For common steel grooved cam pair, set $\mu_{c}=\mu_{f}=0.3, E_{c}=E_{f}=206 \mathrm{GPa}$.

Refer to Fig. 1, radius of curvature of the theoretical profile for grooved cam can be written as follows.

$$
R_{B}=\sqrt{a^{2}+l^{2}-2 a l \cos \left(\theta_{0}+\theta\right)}
$$

\section{Grooved cam profile reconstruction using non-uniform rational B-splines}

Excessive contact stress between grooved cam and oscillating follower can cause unnecessary abrasion of the contact surfaces and distortion of the components. To improve mechanical properties of grooved cam mechanisms, non-uniform rational B-Splines (NURBS) was used to fit motion curves of the oscillating follower.

The NURBS profile of grooved cam is defined as follows.

$$
\theta(\varphi)=\sum_{i=0}^{n} d_{i} R_{i, k}(\varphi)
$$

$$
R_{i, k}(\varphi)=\frac{w_{i} B_{i, k}(\varphi)}{\sum_{i=0}^{n} w_{i} B_{i, k}(\varphi)}
$$

Where, $d_{i}$ is the control point, $w_{i}$ is the factor, $R_{i, k}(u)$ is $k$ rational B-splines, and $B_{i, k}(u)$ is B-splines rational basis function depending on $\boldsymbol{U}=\left[u_{0}, u_{1}, \ldots, u_{n+k+1}\right]$.

Refer to Equation (7) and (8), by facilitating sampling points of the profile, control points and weight factors are calculated and adjusted to optimize NURBS profile. Angular velocity curve $\left(\theta^{\prime}(\varphi)\right)$, Angular acceleration curve $\left(\theta^{\prime \prime}(\varphi)\right)$ and Angular jerk curve $\left(\theta^{\prime \prime \prime}(\varphi)\right)$ are formulated as follows.

$$
\begin{aligned}
& \theta^{\prime}(\varphi)=\sum_{i=0}^{n} d_{i} R_{i, k}^{\prime}(\varphi) \\
& \theta^{\prime \prime}(\varphi)=\sum_{i=0}^{n} d_{i} R_{i, k}^{\prime \prime}(\varphi) \\
& \theta^{\prime \prime \prime}(\varphi)=\sum_{i=0}^{n} d_{i} R_{i, k}^{\prime \prime \prime}(\varphi)
\end{aligned}
$$

Where, $\theta^{\prime}(\varphi), \theta^{\prime \prime}(\varphi), \theta^{\prime \prime \prime}(\varphi)$ represent the 1st-order, the 2nd-order and 3rd-order derivative of rational B-splines respectively. If $3 \leq k \leq 5$, the acceleration curve is continuous, and rigid and flexible impact does not exist in the theory. Furthermore, maximum angular velocity and maximum angular acceleration are reduced by optimizing cam profile with NURBS curve. 


\section{Integrated kinematic and dynamic optimal design}

The peak value of contact stress $\delta_{H \text { max }}$ and inertial forces caused by the peak value of angular acceleration $\theta^{\prime \prime}(\varphi)$ have great influence upon mechanical properties of grooved cam mechanisms. Thinking $d_{i}$ and $w_{i}$ as design variables, integrated kinematic and dynamic optimization mathematical model of grooved cam NURBS profile is expressed as Equation (12).

$$
\min F(\boldsymbol{X})=\left[\delta_{H \max }, \theta_{\max }^{\prime \prime}\right]^{T}
$$

Grooved cam mechanisms do not lock in operation to meet the following constraints.

$$
\begin{aligned}
& \alpha_{\mathrm{s} \max }<[\alpha]_{\mathrm{s}}=35^{\circ} \\
& \alpha_{\mathrm{hmax}}<[\alpha]_{\mathrm{h}}=70^{\circ}
\end{aligned}
$$

Where, $[\alpha]_{\mathrm{S}} 、[\alpha]_{\mathrm{h}}$ are the permissive pressure angle of rise and fall periods respectively, $\alpha_{\mathrm{s} \max } 、 \alpha_{\mathrm{hmax}}$ are also the maximum pressure angle of rise and fall periods respectively.

In order to avoid interference, the minimum radius of curvature $\rho_{\min }$ is limited to Equation (15).

$$
\rho_{\min }-r_{0} \geq 3
$$

Moreover, Grooved cam mechanisms are required to meet the boundary conditions shown in Table 1 .

Table 1. Dwell-Rise-Dwell-Fall kinematics boundary conditions

\begin{tabular}{|l|c|c|}
\hline Cam rotation angle $\varphi /\left(^{\circ}\right)$ & 0 & 150 \\
\hline Follower angular displacement $\theta /\left(^{\circ}\right)$ & 0 & 20 \\
\hline Follower angular velocity $\theta^{\prime}$ & 0 & 0 \\
\hline Follower angular acceleration $\theta^{\prime \prime}$ & 0 & $\neq 0$ \\
\hline Follower Angular jerk $\theta^{\prime \prime \prime}$ & $\neq 0$ & 0 \\
\hline
\end{tabular}

\section{Improved artificial fish swarm algorithm}

Since artificial fish swarm algorithm [9-10] easily fell into local solutions, improved artificial fish swarm algorithm (IAFSA) was proposed by combining the taboo search strategy and variable parameters optimization effectively as shown in Fig. 2.

As shown in Fig. 3, in order to speed up searching, the first IAFSA optimization was performed in early algorithm by setting parameters $P_{l}$ : artificial fish perception range visual, the maximum moving distance step, congestion factor $\delta$ and number of fish $N$ for greater values, feeding frequency Try_number for smaller value. Pareto-optimal solution was locked using taboo criteria, and no Pareto-optimal solution domain was tabooed to avoid the second optimization blindness. In the algorithm later, in order to improve the searching precision, the second IAFSA optimization was carried out by setting parameters $P_{2}$ : visual, step, $\delta$ and $N$ for smaller values, Try_number for larger value. Finally the
Pareto-optimal solution was placed in the bulletin board and outputted.

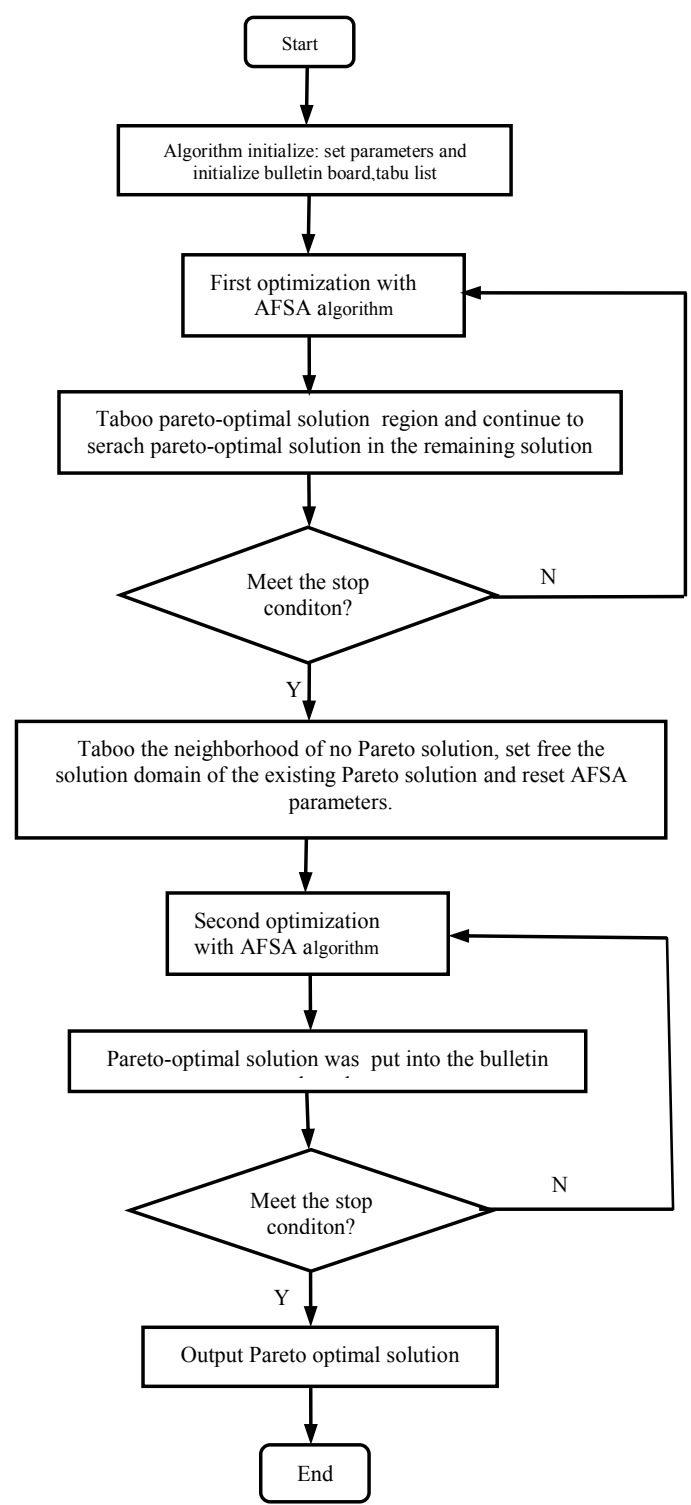

Fig. 2. Flow diagram of Improved Artificial Fish Swarm Algorithm

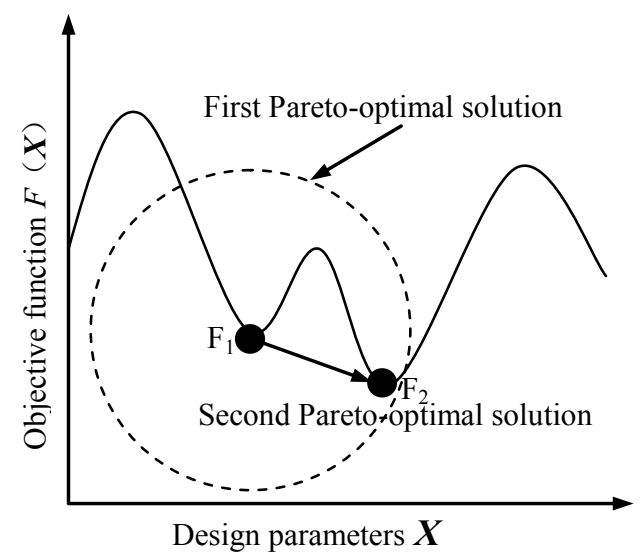

Fig. 3. Optimization twice with variable parameters 


\section{Design example}

The related specifications of the grooved cam mechanism were $T=27 \mathrm{~N} \cdot \mathrm{m}, r_{0}=54 \mathrm{~mm}, l=200 \mathrm{~mm}$, $b=20 \mathrm{~mm}, a=300 \mathrm{~mm}, \quad r_{b}=135, f=0.05, \rho_{1}=0.5$, and $\rho_{2}=1$. Of the follower motion curves, modified sine (MS) curve has low velocity and acceleration, which is suitable for improving mechanical properties of grooved cam mechanism. MS profile was reconstructed using 5order NURBS curve, $n=20$. IAFSA optimization algorithm parameters were set as follows.

Each artificial fish state $\boldsymbol{X}=\left(d_{0}, w_{0}, d_{1}, w_{1}, \ldots d_{\mathrm{n}}, w_{n}\right)$, current food concentration $\min F(\boldsymbol{X})=\left[\delta_{H \max }, \theta_{\max }^{\prime \prime}\right]^{T}$, taboo table length $L=50, N=150$, maximum number of iterations $G m=500, P_{1}$ : visual $=5$, step $=2, \delta=3$, and Try_number $=1, \quad P_{2}: \quad$ visual $=1, \quad$ step $=0.5, \quad \delta=1 \quad$ and Try number $=5$. One of the Pareto-optimal solutions was obtained as shown in Table 2, and mechanical properties comparison between MS curve and NURBS curve have been shown in Fig. 4-Fig. 8.

Table 2. Mechanical properties of MS and NURBS curve

\begin{tabular}{|c|c|c|c|c|}
\hline $\begin{array}{c}\text { Mechanical } \\
\text { properties }\end{array}$ & $\theta^{\prime}{ }_{\max }$ & $\theta_{\max }^{\prime \prime}$ & $\theta_{\max }^{\prime \prime \prime}$ & $\delta_{\text {Hmax }}$ \\
\hline Single stop MS & 0.221 & 0.0047 & 0.00039 & 98.8637 \\
\hline NURBS & 0.223 & 0.0045 & 0.00021 & 93.7658 \\
\hline
\end{tabular}

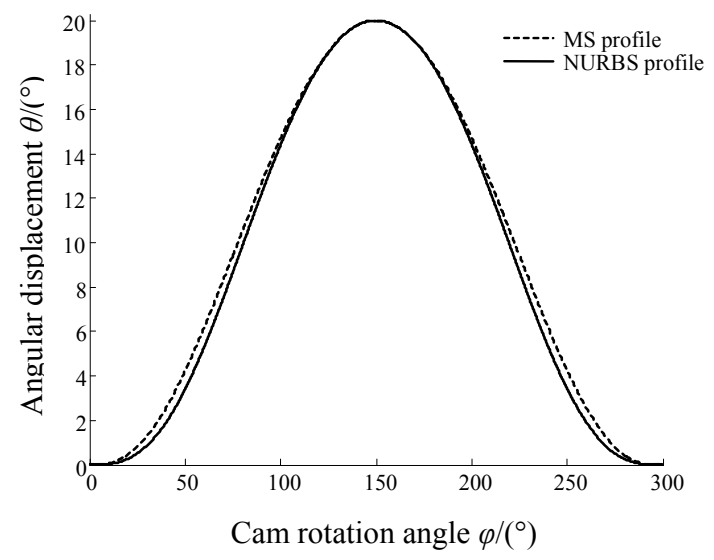

Fig. 4. Angular displacement curve

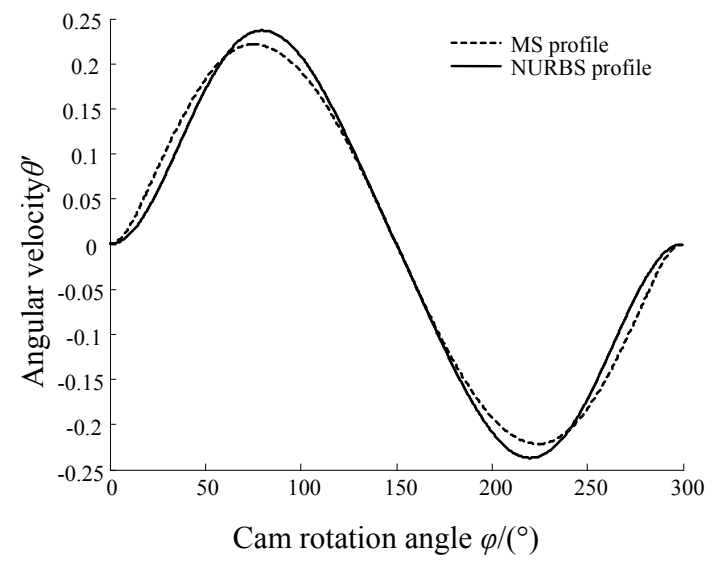

Fig. 5. Angular velocity curve

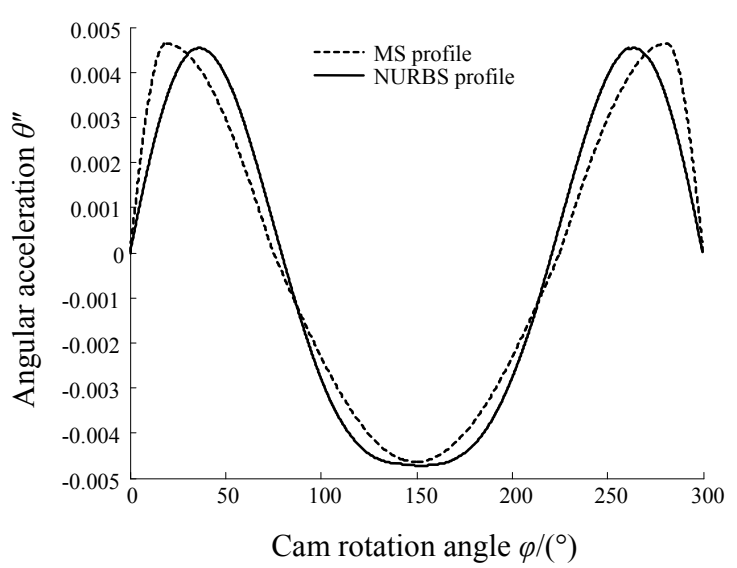

Fig. 6. Angular acceleration curve

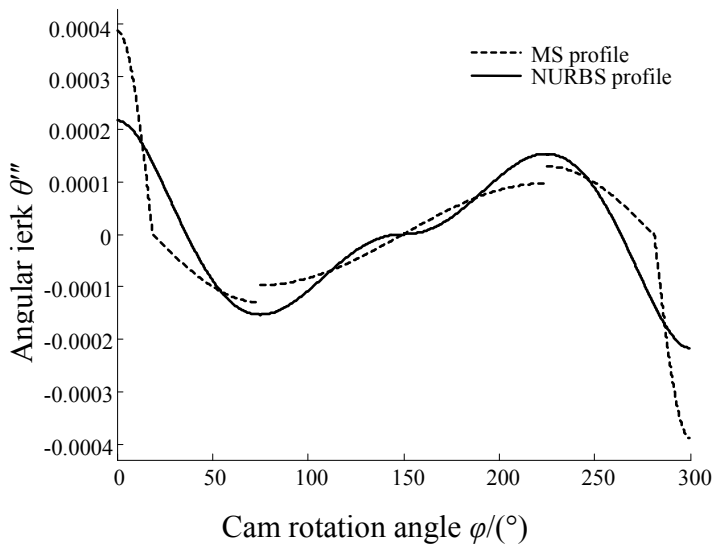

Fig. 7. Angular jerk curve

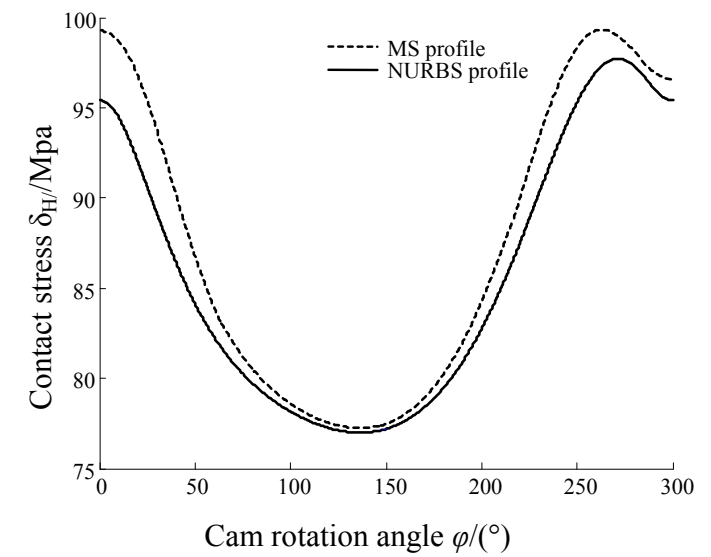

Fig. 8. Contact stress curve

It shows the motion curve of the follower is smoother by applying NURBS curve to optimize MS profile. Especially angular jerk curve is continuous, and the peak value of angular jerk is smaller in comparison with MS profiles. Fig. 6 also illustrates, the peak value of angular acceleration curve is decreased from 0.0047 to 0.0045 by 4.3\%. Meanwhile, the contact stress between grooved cam and follower is decreased from $98.8637 \mathrm{Mpa}$ to $93.7658 \mathrm{Mpa}$ by $5.16 \%$ in Fig. 8. For grooved cam mechanisms, kinematic and dynamic characteristics of NURBS profile have been greatly improved compared with MS curve. With NURBS profile applied, shock and wear in grooved cam mechanisms are reduced, which improves the working reliability of grooved cam mechanisms, extends the service life. 


\section{Conclusions}

Dynamic analysis was first carried out to provide the expression for the contact stress in grooved cam mechanisms. By adjusting the node vector, control points and power factors, grooved cam profile was reconstructed with NURBS curve. Integrated optimum design model which decreased the maximum contact stress and angular acceleration was developed sequentially. A design example was demonstrated in the end. The design results indicate that the peak value of angular acceleration and contact stress can be effectively reduced, which improve mechanical properties of grooved cam mechanism to some extent.

\section{Acknowledgements}

We would like to thank "National Natural Science Foundation of China (Nos.31671632, 31701325)", "Natural Science Foundation of Shandong Province (BS2015NY012)" and "Key Research and Development Program of Shandong Province (2016GNC112007)" for financial supports.

\section{References}

1. Demeulenaere, B. and De Schutter, J., Input torque balancing using an inverted Cam mechanism, ASME transactions, Journal of Mechanical Design, 127 (2003).

2. Demeulenaere, B., Spaepen, P., and De Schutter, J., Input torque balancing using a Cam-based Centrifugal Pendulum:Design procedure and Example, Journal of Sound and Vibration 283, 1-2 (2005).

3. Nayak Nagaraj, Lakshminarayanan P.A., Babu M.K. Predictions of cam follower wear in diesel engines, Wear, 2006.

4. Chen F. Y., Mechanics and Design of Cam Mechanisms, Pergamon Press, New York, 1982.

5. Tsay D. M., Huey C. O., Cam motion synthesis using spline function", Trans. ASME, J. of Mech., Trans., Auto. in Des. Il0, 2 (1988).

6. Yoon K., Rao S. S., Cam motion synthesis using cubie spline, Trans. ASME, J. of Mech. Des. 115(3), 3 (1993).

7. Guantao Xuan, Yuanyuan Shao, Zhaoqin Lü, Profile Reconstruction of Spatial Indexing Cam and Simulation Based on NURBS, Transactions of the Chinese Society for Agricultural Machinery 43, 6 (2012).

8. Rongyu Ge, Shiwei Li, Li Liu, Flexible cam curve expressed by NURBS and mul-tiobjective Optimization based on genetic algorithm, Transactions of the Chinese Society for Agricultural Machinery 39, 2 (2008).

9. Na Fang, Jianzhong Zhou, Rui Zhang, A hybrid of real coded genetic algorithm and artificial fish swarm algorithm for short-term optimal hydrothermal scheduling, International Journal of Electrical Power \& Energy Systems, 62(2014).

10. X. Li, Z. Shao, J. Qian, An optimizing method based on autonomous animats: fish-swarm algorithm, Syst Eng Theory Pract 22, 11(2002). 